\title{
Buenas prácticas cooperativas en Cuba
}

\author{
Lien Soto Alemán¹, José Manuel Figueroa González²
}

Sumario: I. Introducción. II. Buenas prácticas y responsabilidad social: un vínculo estrecho. III. Experiencias de buenas prácticas cooperativas en Cuba. IV. Ideas conclusivas. Bibliografía.

Resumen: La sociedad actual se muestra cada vez más exigente con el tejido empresarial, en términos de que adopten buenas prácticas, y asuman una responsabilidad social que supere la dimensión estrictamente económica. En el ámbito cooperativo la responsabilidad social se percibe como una alternativa que enaltece la ética y la filosofía cooperativa, en la práctica y cumplimiento de los principios y valores de estas; se ha elevado a rango de principio en algunas legislaciones como la cubana. En el presente artículo se muestran algunos ejemplos de buenas prácticas cooperativas en Cuba, y se analiza la necesidad de medir y evaluar el impacto social de estas organizaciones. Se concluye con reflexiones en torno a la necesidad de regular el Balance Social Cooperativo en Cuba.

Palabras clave: Cooperativa, responsabilidad social, buenas prácticas, balance social.

Abstract: In the present time, the society show itself each time more capable with enterprises net, in terms that they to assume good practices, and adopted a social responsibility wish not only are not in the economical dimension. In the cooperative environment, the social responsibility is perceived like an alternative that ennobles the ethics and the cooperative philosophy, in the practice and fulfillment of its principles and values. In some legislations, like the cuban, has risen to the range of a principle. Currently article show some examples of goods cooperatives practices, and analyzed the need of measure and evaluate the social impact of this organizations. The present article con-

1 Máster en Derecho de la Economía. Profesora Auxiliar de la Facultad de Derecho de la Universidad de La Habana. Miembro de la Asociación Internacional de Derecho Cooperativo con sede en la Universidad de Deusto, Bilbao. E-mail: lien@lex.uh.cu

2 Doctor en Ciencias. Director del International Center for entrepreneur de BarceIona (ICEB), España. E-mail: jfigueroa@iceb-edu.com 
cluding with reflections around the need the Cooperative Social Balance in the Cuban Legislation.

Keywords: Cooperative, social responsibility, good practices, social balance. 


\section{Introducción}

El movimiento cooperativo cubano actualmente se integra por las cooperativas agropecuarias, en sus dos formas, las de producción y las de créditos y servicios, consignadas desde la Ley de Reforma Agraria del 17 de mayo de 1959. El marco regulatorio está contenido en el Decreto Ley No. 365 «De las Cooperativas Agropecuarias» y el Decreto No. 354 «Reglamento de las Cooperativas Agropecuarias»3. A su vez, las cooperativas del sector no agropecuario son reguladas por el Decreto Ley No. 366 «De las Cooperativas no Agropecuarias» y el Decreto No. 356 «Reglamento de las Cooperativas no Agropecuarias ${ }^{4}$. Comparten espacio, aunque con una realidad jurídica diferente, las Unidades Básicas de Producción Cooperativa (UBPC), las que constituyen un patrimonio con personalidad jurídica, en este caso compuesto por los bienes agropecuarios y los frutos de la tierra recibida en usufructo, tierra que se mantiene bajo la propiedad del Estado 5 .

En el marco de la actualización del modelo económico cubano, se asume el papel complementario del sector cooperativo respecto de la empresa estatal6; y se entiende que las acciones a desarrollar por las cooperativas están dirigidas a potenciar el desarrollo local del entorno

3 Publicadas en la Gaceta Oficial Ordinaria (GOO) No. 37, de 24 de mayo de 2019. Esta norma derogó la Ley No. 95 de fecha 2 de noviembre de 2002, «De las Cooperativas de Producción Agropecuaria y de Créditos y Servicios». Publicada en GOO No. 72, de 29 de noviembre de 2002.

4 Publicadas en la GOO No. 63 de 30 de agosto de 2019. Estas normas derogaron el Decreto Ley 305 "De las cooperativas no agropecuarias» y el Decreto 309/12, "Reglamento de las cooperativas de primer grado», Publicadas en GOO No. 15, de 20 de marzo de 2013.

5 Reguladas en el Decreto Ley No. 365 «De las Cooperativas Agropecuarias» (arts. 5, 12, 33, 40, 47, 48), op. cit. Esta norma en su DISPOSICIÓN FINAL PRIMERA, deroga la norma precedente que regulaba estas figuras: el Decreto Ley No. 142, de 20 de septiembre de 1993, "Sobre las Unidades Básicas de Producción Cooperativa». Publicada en Gaceta Oficial Extraordinaria (GOE) No. 6, de 21 de septiembre de 1993.

6 Lineamiento 3-«El Modelo Económico y Social Cubano consolida la propiedad socialista de todo el pueblo sobre los medios de producción fundamentales como la forma principal en la economía nacional. Además, reconoce en las actividades que se autoricen la propiedad cooperativa, la mixta, la privada de personas naturales o jurídicas cubanas o totalmente extranjeras, de organizaciones políticas, de masas, sociales y otras entidades de la sociedad civil. Todas funcionan e interactúan en beneficio de la economía y están sujetas al marco regulatorio y de control definido por el Estado».

Lineamiento 17-«lmpulsar el desarrollo de los territorios a partir de la estrategia del país, de modo que se fortalezcan los municipios como instancia fundamental, con la autonomía necesaria, sustentables, con una sólida base económico-productiva, y se reduzcan las principales desproporciones entre estos, aprovechando sus potencialidades. Elaborar el marco jurídico correspondiente». 
donde insertan su gestión. Entendido el territorio no como espacio abstracto e indiferenciado sino como actor fundamental de desarrollo, integrado no sólo por el medio físico sino por los actores sociales y sus organizaciones, las instituciones locales; los cuales, además del capital económico y financiero incorporan las diferentes dimensiones del capital humano, el capital social e institucional y el capital natural ${ }^{7}$. Es por ello que el tejido económico en la base tiene en las cooperativas cubanas actores fundamentales y de ellas se espera una mayor responsabilidad social.

Partiendo del análisis de la situación actual de las cooperativas cubanas, expuesto en los artículos anteriores, el presente trabajo persigue, como objetivo esencial, exponer algunos ejemplos de buenas prácticas cooperativas en Cuba, así como, argumentar con elementos teóricos y prácticos la necesidad de fomentar los comportamientos responsables en estas organizaciones, desde una perspectiva que persigue reafirmar la necesidad de contar con instrumentos de medición de la incidencia social de las cooperativas, en todas sus vertientes.

Los resultados que se presentan se basan en un tipo de investigación empírica. Para el levantamiento de la información constituyen fuente primaria, además, la revisión documental y la observación. Se desarrollaron dinámicas grupales con las Juntas Directivas y los asociados; así como, en el caso particular de la cooperativa de reciclaje de materias primas de San José de Las Lajas, con los actores comunitarios tales como: representante de la Organización de Pioneros José Martí (OPJM), el delegado del Poder Popular, el delegado municipal del Ministerio de Industrias, un representante de la empresa, y la federación. Estas sesiones tuvieron lugar a fin de caracterizar a la cooperativa, y conocer los desafíos que tienen como organización en el contexto económico, político y socio cultural en el que se insertan, así como los factores que garantizan su éxito ${ }^{8}$. Fueron consultadas, además, fuentes bibliográficas nacionales, que abordan la temática en análisis.

Partido Comunista de Cuba (VII Congreso): Actualización de los Lineamientos de la Política Económica y Social del Partido y la Revolución para el período 2016-2021, abril de 2016. Recuperado de http://www.cubadebate.cu/especiales/2016/09/13/vea-el-texto-integro-de-la-actualizacion-de-los-lineamientos-para-el-periodo-2016-2021-pdf/ Consultado el 2 de octubre de 2016.

7 Vid. Francisco Albuquerque Llorens: «Desarrollo económico local y descentralización en América Latina», Revista de la CEPAL, No. 82, 2004, p. 22.

8 Información obtenida de trabajo investigativo anterior donde participó la autora en coautoría. En ese sentido, Vid. Soto Alemán, L.; Llanes Guerra, O. y González Ferrer, Y.: «El Balance Social Cooperativo en Cuba: Estado de la cuestión». En Schujman, M.; Iturraspe Oviedo, F.; Fajardo García, I. y Chaves Gaudio, R. (Coords): La Participación de 


\section{Buenas prácticas y responsabilidad social: un vínculo estrecho}

Las concepciones en torno a la Responsabilidad Social Empresarial (RSE) datan de principios del siglo $x X$, aunque las bases conceptuales son mucho más antiguas, siendo tradicionalmente reconocido el vínculo entre la sociedad y la empresa mediante la filantropía —una acción de caridad de la empresa hacia su comunidad — 9 . No es hasta mediados del siglo xx que este fenómeno acentúa su presencia en el sector empresarial, dada la proliferación de temas relevantes como los derechos laborales, la protección al consumidor y los problemas ambientales.

Actualmente la RSE es concebida como una nueva forma de hacer negocios, donde el objetivo estratégico de la empresa no solo se enfoca en la generación de utilidades y el rendimiento económico de ésta, sino en la búsqueda de valor para todos los grupos de interés (teoría stakeholders ${ }^{10}$ ) con los cuales interactúa en su gestión, desde una triple dimensión: económico, social y ambiental sostenible ${ }^{11}$. Social, a partir de la articulación de intereses económicos con intereses sociales de la comunidad donde inserta su gestión el sujeto, laboral, mediante las Políticas de empleo, Seguridad y salud en el trabajo, capacitación y educación; así como, ambiental, mediante la prevención de riesgos ambientales, mitigación de daños, actitudes proactivas ante el cuidado del ambiente, formación y educación, consumo responsable, entre otras ${ }^{12}$.

los asociados en las entidades de la economía social y solidaria. Cooperativas de trabajo asociado. Autogestión. Carta del trabajador autogestionario, 1a ed. Rosario: UNR Editora. Editorial de la Universidad Nacional de Rosario, 2019.

9 No se debe confundir la filantropía con la responsabilidad social por cuanto las actividades filantrópicas por sí mismas no consiguen el objetivo de integrar la responsabilidad social en la organización; en ello ha insistido la Norma ISO 26000, que será abordada más adelante. Incluso la Filantropía y la caridad son términos divergentes.

10 De acuerdo con Freeman son: " cualquier grupo o individuo que puede afectar o ser afectado por la consecución de los objetivos de la empresa». Vid. Freeman R.E.: Strategic management: a stakeholders approach, en Pitman press, Boston, 1984, p. 229.

11 Vaca Acosta et al.: "Análisis de la Responsabilidad Social Corporativa desde tres enfoques; stakeholders, capital intelectual y teoría institucional», Revista Conocimiento, innovación y emprendedores; camino al futuro. Vol. 3. Universidad de La Rioja, España, p. 18, Disponible en: <http//: dialnet.unirioja.es/descarga/articulo/2234835.pdf>. Fecha de consulta: 5 de mayo de 2016.

12 Desde los años 80 se asistió a una creciente conciencia pública en relación al papel de los negocios en la sociedad tras desastres medioambientales como Chernobyl y Exxon Valdez. Para los años 90, el Informe Brundtland (1987) y la Cumbre de Rio (1992) inspiraron un concepto de desarrollo más allá del crecimiento económico y de la continua generación de riqueza definida exclusivamente en términos financieros para englo- 
La norma ISO 26000:2010 "Guidance on Social Responsibility», proporciona una guía para todo tipo de organizaciones, tanto en los sectores público como privado, en los países en desarrollo y en los desarrollados, así como, en las economías en transición, sobre temas relacionados con la responsabilidad social. La norma plantea como objetivo esencial de esta, que las organizaciones tomen responsabilidad ante los impactos que sus decisiones y actividades ocasionan en la sociedad y en el medio ambiente, mediante un comportamiento ético y transparente. Establece como principios básicos los siguientes: responsabilidad; transparencia; ética; enfoque multistakeholder; respeto a leyes y normas; respeto a normas internacionales de comportamiento; y respeto a los derechos humanos ${ }^{13}$.

Estas nociones sobre la RSE parten de asumir la esencia social de las organizaciones empresariales como conjunto de personas que interactúan en el marco de la sociedad, tanto desde un punto de vista de su propia composición elemental como desde la óptica de miembro integrante de un sistema social, de ahí su importancia. Ahora bien, la RSE implica rendición de cuentas a los grupos de interés, a la empresa no debe bastarle decir que es socialmente responsable sino debe demostrarlo, el interés por esto es creciente en los últimos años, en tal sentido, el impacto social de las actividades de la empresa debe ser medible y estar contenida en un documento de alcance público. Para presentar esta información, se han trabajado diferentes modelos que facilitan de una parte la intraevaluación de las empresas, es decir a lo interno se posibilita la autoevaluación de organización y el establecimiento de estrategias y metas de desempeño, a lo externo posibilita la interevaluación de las empresas por los organismos evaluadores y la comparación de los datos ofrecidos por empresas diferentes. Este documento es conocido como Balance Social.

Aunque el concepto de RSE nace en el sector empresarial, sus principios se alinean con los principios y valores que impulsan el quehacer de las cooperativas.

Desde esta perspectiva, es de apreciar que las nociones conceptuales sobre la RSE adquieren una connotación particular en el ámbito

bar conceptos vinculados a la calidad de vida. Se manejaba el término desarrollo sostenible como el desarrollo que satisfacía las necesidades de las generaciones presentes sin comprometer las posibilidades de las del futuro para atender sus propias necesidades.

13 ISO-Organización Internacional de Normalización-Norma Internacional ISO 26000, Guía de Responsabilidad Social: Primera Edición, Ginebra, 2010. Disponible en http://www.iso.org/iso/iso_26000_project_overview-es.pdf. Fecha de consulta: 12 de noviembre de 2016. 
cooperativo y se configura como un aspecto intrínseco a la propia naturaleza de estas organizaciones. Es así que, la conceptualización de la Responsabilidad Social Cooperativa (RSCoop) se enmarca en el cumplimiento de los valores y principios ${ }^{14}$, genuinos y esenciales, del cooperativismo como movimiento y forma parte de su razón de ser. En otras palabras, los principios y valores que constituyen la identidad de la cooperativa representan los pilares sobre los cuales se erige la responsabilidad social de estas organizaciones ${ }^{15}$.

Para BASTIDAS DELGADO «la responsabilidad social de las cooperativas forma parte consustancial de la misión de la organización, pues ellas son constituidas para satisfacer las necesidades de sus asociados a través de la actividad asignada y no necesariamente a través de aumento de capital, lo que se traduce en aprovechar al máximo los siempre limitados recursos de las cooperativas, para obtener el máximo de satisfacción en lo social» ${ }^{16}$.

Nótese en la visión del autor el refuerzo de la dimensión económica de los recursos de la cooperativa siempre en función de la satisfacción de aspectos sociales. Es lógico, las cooperativas aún sin practicar el ánimo de lucro requieren sobrevivir y desarrollarse en espacios de mercado, es lo que ALFONSO ALEMÁN et al. denominan soporte

14 Se podría entender por identidad cooperativa el conjunto de principios y valores universalmente reconocidos, que expresan la dimensión social y económica de la cooperativa como forma de organización, funcionamiento de esta entidad en la sociedad.

Este binomio «económico-social» que describe el fin de una cooperativa, obedece de una parte a la necesidad de su sobrevivencia en la economía actual y al hecho cierto de que debe responder a determinados estándares de eficiencia y productividad para poder desarrollarse, y en esa misma medida lograr el bienestar social, de sus asociados, de la comunidad donde se inserta y de la sociedad en sentido general a la cual pertenece (el fin social que persigue). Vid. Soto AlemÁn, L.: Presupuestos Teórico-Jurídicos del Balance Social Cooperativo en Cuba, Tesis en opción al grado científico de Master en Derecho de la Economía, La Habana, 2016, p. 25.

Este criterio se comparte por otros investigadores cubanos. Vid. Rodríguez MusA, O.: Presupuestos Teóricos para el redimensionamiento constitucional de la cooperativa en Cuba, Tesis en opción al grado científico de Doctor en Ciencias Jurídicas, La Habana, 2016, p. 26.

15 Principios cooperativos: Asociación voluntaria y abierta; Control democrático por los asociados; Participación económica de los asociados; Autonomía e independencia; Educación, capacitación e información; Cooperación entre cooperativas; e, Interés por la comunidad. Alianza Cooperativa Internacional: «Declaración sobre la Identidad Cooperativa», adoptada en el Congreso del Centenario en Manchester, 23 de septiembre de 1995. Disponible en: www.idelcoop.org.ar/sites/default/files/revista/articulos/pdf/... Fecha de consulta: 20 de octubre de 2014.

16 Bastidas Delgado, O.: "La especificidad cooperativa», Universidad Central de Venezuela, Caracas, 2004, p. 2 a 19. 
materia/17, lo que significa que las empresas cooperativas deberán asegurar el cumplimiento de su compromiso social, sobre la base de su desempeño económico y financiero, a partir de recursos propios generados como excedentes de su gestión económica, así como de otros que se obtengan como resultado de asignaciones estatales con este fin o de gestiones específicas con otras instituciones y organismos, por ello constituye una condición básica y al mismo tiempo limitante de la Responsabilidad Social Cooperativa ${ }^{18}$.

El Decreto Ley No. 365 contempla en su artículo 8, inciso k), el principio de responsabilidad social y contribución al desarrollo de la economía nacional en términos de que «los planes y programas de las cooperativas agropecuarias tienen como objetivo fundamental contribuir al desarrollo económico y social sostenible de las comunidades en que están enclavadas, del municipio y del país en general, en armonía con el medio ambiente... ${ }^{19}$.

En ese sentido el legislador cubano elevó a rango de principio la responsabilidad social en el ámbito cooperativo, aunque ciertamente, a pesar de que fija las pautas esenciales, a manera de reconocimiento de la responsabilidad social como principio, no aborda concretamente las expresiones de esta ni cómo medirlas o evaluarlas.

El Decreto Ley No. 366, al igual que la norma homóloga del sector agropecuario, contempla en su artículo 6, inciso f) el principio de Responsabilidad Social, contribución al desarrollo planificado de la economía y al bienestar de sus socios y familiares en virtud del cual los planes de las cooperativas tienen como objetivo contribuir al desarrollo económico y social sostenible de la nación desde el ámbito territorial donde realiza sus actividades, proteger el medio ambiente, desarrollar sus actividades sin ánimo especulativo y garantizar el cumplimiento disciplinado de las obligaciones fiscales y otras ${ }^{20}$.

En efecto, un desarrollo sostenible requiere de la utilización eficaz y eficiente de los recursos económicos, sociales y medioambientales, sin comprometer los recursos futuros, lo que demanda de cooperación en el tejido organizacional por medio de la responsabilidad so-

17 Alfonso-Alemán J.L.; Rivera-Rodríguez C. A.; Labrador-Machín O.: «Responsabilidad social empresarial. Sus particularidades en las empresas cooperativas», Revista Avances, CIGET Pinar del Rio, Vol. 11, No. 1, marzo 2009, p. 11.

18 Rivera-Rodríguez C.A.; Labrador-Machín O.; Alfonso-Alemán J.L: «Retos del cooperativismo como alternativa de desarrollo ante la crisis global. Su papel en el modelo económico cubano». En Piñeiro Harnecker, C. (comp): Cooperativas y Socialismo: una mirada desde Cuba, Editorial Caminos, La Habana, 2011, p. 401.

19 Cfr. Decreto Ley No. 365, op. Cit., p. 557.

20 Decreto Ley No. 366 «De las Cooperativas no Agropecuarias», op. cit., p. 1334. 
cial. De manera que las buenas prácticas no son sólo internas a las cooperativas, sino que reflejan cómo estas actúan dentro de un contexto determinado buscando preservar la identidad de estas organizaciones.

Las buenas prácticas cooperativas son una respuesta a la implementación de iniciativas que contribuyen a minimizar los impactos negativos generados por la actividad de la cooperativa y a potenciar todas las acciones que contribuyan con impactos positivos. Son criterios que permiten desarrollar herramientas para ejecutar un proceso o una operación, contribuyen a que la organización alcance un alto desempeño social y ambiental y les sirve como meta para lograr niveles de excelencia. Se sustenta en procesos que son propios para cada cooperativa, que les permite crecer y cambiar respondiendo a su propio interés, capacidad, y responsabilidad social ${ }^{21}$. En el sector cooperativo las buenas prácticas constituyen expresión de su responsabilidad social.

Muchas veces las cooperativas no tienen una conciencia clara de que por su naturaleza ejecutan buenas prácticas de responsabilidad social porque no están establecidos programas que lo orienten y controlen o no queda debidamente instrumentado el "cómo» hacia lo interno de su organización. Con independencia de ello, es notable que en Cuba, las cooperativas de diferentes sectores, asumen cada vez más buenas prácticas cooperativas. A renglón seguido se analizan algunas de ellas.

\section{Experiencias de buenas prácticas cooperativas en Cuba}

Para el levantamiento de la información, además de la revisión documental, se realizaron indagaciones directas en cooperativas de cinco sectores esenciales (agropecuarias, 25; transporte, 4; construcción, 12; gastronomía, 21; servicios, 8; construcción, 12, y, reciclaje de materias primas, 2), radicadas, en lo fundamental, en La Habana y en provincias del occidente del país ${ }^{22}$. En relación al procesamiento de la información, fueron identificadas las siguientes variables: conocimiento sobre los principios cooperativos y particularmente sobre la Responsabilidad Social Cooperativa (RSCoop); participación de los socios en la dirección y gestión administrativa de la cooperativa; relaciones de la cooperativa

21 Montenegro Morales., M.: «Experiencias de buenas prácticas en la Cooperativa No Agropecuaria de Servicios de Construcción, Andamios y Cofres», COODES, Revista de Cooperativismo y Desarrollo, Volumen No. 6 No. 1, enero-junio, 2018, p. 111.

22 Matanzas, Mayabeque y Pinar del Río. 
con los socios y sus familiares; realización de acciones de mitigación de daño ambiental por las actividades de la cooperativa; y, vínculo o impacto de la cooperativa con la comunidad.

\section{a) Conocimiento sobre los principios cooperativos y particularmente sobre la Responsabilidad Social Cooperativa}

El principio de «Educación, capacitación e información», conocido como «el principio de principios», busca situar al ser humano en el epicentro de la actividad empresarial de la cooperativa, ya que en estas se hace imprescindible que sus miembros reciban una apropiada formación que le permita conocer qué es una cooperativa, cómo funciona, qué derechos y deberes tienen, entre otras cuestiones medulares. Para ello se valen de un fondo educacional que permite lograr de manera efectiva dicha preparación que posibilita una mejor participación en la gestión y administración. En este sentido es importante señalar, como afirma RODRÍGUEZ MUSA, que «la educación cooperativa no se agota con la mera instrucción, sino que debe proporcionar conocimientos acerca de los principios y métodos cooperativos; debe inducir a los asociados a participar activamente en su cooperativa, a deliberar correctamente en las asambleas, a elegir conscientemente a sus autoridades y a controlar su actuación; debe enseñar a los dirigentes a orientar y expandir las actividades comunes, aspectos sin los cuales se dificulta el ejercicio consciente de la participación y el control en las múltiples esferas en que la cooperativa puede propiciarlos» 23 .

Desde esta perspectiva, el trabajo indagatorio dirigido a conocer el conocimiento por parte de los socios sobre los principios cooperativos y, particularmente, la RSCoop demostró que, de manera general, existe diversidad de opiniones sobre el tema, con tendencia al desconocimiento. Tras el análisis de los resultados de las entrevistas realizadas, se constató que en cuanto a los principios que informan al sistema cooperativo, los socios que integran las cooperativas entrevistadas no lo conocen, aunque en algunos casos si bien no con el nomen que establece la ley, sí el contenido que informan a estos principios; haciendo gran énfasis cada uno sobre la autonomía, la participación económica de los socios, la educación, el control democrático de los miembros y la cooperación entre cooperativas.

23 Rodríguez Musa, O.: La Cooperativa como figura jurídica. Editorial DYKINSON, S.L., Madrid 2011, p. 37. 
En relación a la RSCoop, solo el $15 \%$ la concibe como una forma de actuación contributiva de la cooperativa hacia la sociedad y el medio ambiente, que va más allá del cumplimiento de los objetivos económicos de la misma, por tanto, se acercan a otras esferas como la social y la medioambiental, pero con un limitado alcance. Ello, refleja insuficiencias en los proceso de formación de los miembros de la cooperativa, que guarda relación con sus contenidos así como con la sistematicidad de los mismos. Es importante destacar la labor que realiza la Cooperativa Allen y Asociados, en base al principio de Educación - Información y Entrenamiento con los cursos de verano que en ella se imparten. También, la cooperativa "Gesin», que tiene firmado un «Convenio de Colaboración» con el Centro de Estudios de Técnicas de Dirección (CETED) de la Universidad de La Habana que entre sus objetivos plantea:

- La realización conjunta de proyectos de investigación sobre métodos y estilos de dirección, comportamiento organizacional y evolución del sector cooperativo en Cuba.

- Facilitar la inserción de socios de Gesin en acciones de capacitación facilitadas por el CETED o por otros actores donde aquellos puedan insertarse.

- Participación de socios de la cooperativa en cursos y talleres posgraduados.

- Participación de socios de Gesin en proyectos de desarrollo docente y fortalecimiento institucional ejecutado por el CETED, brindando apoyo material y financiero, en correspondencia con las reservas patrimoniales de la cooperativa, destinada a estos efectos.

También, esta cooperativa tiene suscrito un »Convenio de Colaboración» con la Universidad de Pinar del Río (UPR), que tiene por objeto el compromiso de estos sujetos en promover y desarrollar, en el marco de sus posibilidades, aquella actividades de complementación y colaboración que permita la realización de programas conjuntos de investigación, docencia y extensión universitaria, a fin de materializar la responsabilidad social inherente a ambas instituciones, sobre todo en torno a la «Educación Cooperativa».

Otros ejemplos los encontramos, en los encuentros programados entre las cooperativas de servicios automotores de la Habana, que persiguen una retroalimentación técnica de la labor que realizan, a través de intercambios que realizan con cierta sistematicidad. Ello refleja además preocupación por el cumplimiento del principio de 
«cooperación entre cooperativas» ${ }^{24}$. En otro orden, se pudo constatar la presencia de reservas para educación y formación cooperativa en varias cooperativas del sector no agropecuario del país, en reflejo de la necesidad de asumir de manera consciente esta planificación en las organizaciones.

Si la educación cooperativa se asimila al interior de estas organizaciones, y de los organismos estatales mandatados a asumir su fomento, se estaría dando inicio a un proceso de fortalecimiento y aprendizaje que contribuiría a robustecer el ideal cooperativo en el país.

b) Participación de los socios en la dirección y gestión administrativa de la cooperativa

En relación al principio de "Control democrático por los asociados», en las Cooperativas de base los miembros tienen igual derecho de voto, es decir un miembro, un voto, esta constituye la regla de oro de la democracia cooperativa. En tal sentido, todos los asociados tienen un derecho igualitario a participar en la dirección y administración de la empresa y pueden ser elegidos para esos cargos. Como afirma RODRIGUEZ MUSA «en las cooperativas, el poder de decisión tiene un carácter intuito personae, es decir, depende de las personas en sí mismas, para lo cual se establece que todos sus miembros poseen iguales derechos; igualdad que se evidencia en el voto igualitario para la toma de decisiones, en el derecho de todos a elegir y ser elegidos para ocupar los cargos directivos y en el aprovechamiento común de las ventajas que proporciona la empresa; además de que los representantes son responsables de sus acciones ante los socios, desde el momento de su elección y durante todo su mandato» 25 .

El capital humano de una cooperativa es su recurso más valioso, y en un sistema como el nuestro con mayor razón se fundamenta una actuación proactiva en pos del bienestar de los socios, a fin de que se sientan partícipes del proceso de gestión y producción de la cooperativa. Desde esta perspectiva, el que los asociados sean trabajadores y dueños de la cooperativa es una situación realmente diferente a otras organizaciones. Al indagar sobre la participación de los socios en la di-

24 Artículo 6, g) colaboración y cooperación entre cooperativas y otras entidades: las cooperativas se relacionan entre sí y con otras entidades, estatales o no, mediante contratos, convenios de colaboración, intercambio de experiencias y otras actividades lícitas. Vid. Decreto Ley No. 366 «De las Cooperativas no Agropecuarias», op. cit., p. 1335.

25 Rodríguez Musa, O.: La Cooperativa como figura jurídica, op. cit., p. 35 y ss. 
rección y gestión administrativa de la organización, se hizo énfasis en el trabajo que se realiza con los socios, previo a su incorporación a la cooperativa, así como al sentido de pertenencia que se logra una vez incorporados a esta.

Los espacios de participación referidos en los Decretos-Leyes son las reuniones de la Asamblea General ${ }^{26}$, de ahí que sean espacios trascendentales para el fortalecimiento del principio cooperativo de control democrático. En general, las asambleas generales ordinarias de las cooperativa se realizan según lo establecido en estatutos, asimismo, se convocan tantas asambleas extraordinarias como sea necesario ${ }^{27}$. Los resultados de los cómputos de votación realizados por los socios para la elección de los órganos colegiados y de dirección se dan conocer inmediatamente a la asamblea.

Debido a la importancia económica y medioambiental que implica la recuperación y procesamiento de desechos reciclables, es que se han adoptado medidas que permitan a corto y mediano plazo incrementar la recuperación de todo lo reciclable, en este marco tuvo lugar la creación experimental de 15 cooperativas no agropecuarias de reciclaje, lo que representa el 4,3\% del total existente en marzo 2017. Es en este contexto que se crea la Cooperativa de Reciclaje de Desechos de San José de las Lajas, ubicada en la provincia de Mayabeque, en el municipio de San José de las Lajas. Es una cooperativa no agropecuaria (CNoA) que goza de un alto reconocimiento por su gestión, por lo que fue condecorada Vanguardia Nacional en el año 2017.

En esta CNoA se reconocen como elementos de buenas prácticas en su gestión: la distribución equitativa de tareas sin mirar cargos o profesiones, que permite un mejor aprovechamiento de la fuerza de trabajo; control más efectivo y mayor interés de todos los socios por éste; colectivo integrado y con elevada experiencia en el reciclaje; alto sentido de pertenencia y grado de compromiso de los socios. En general, se refuerzan valores tales como ayuda mutua, responsabilidad, democracia, equidad, transparencia, sentido colectivista, conciencia ambiental.

26 Artículo 27.1. Decreto Ley No. 366 «De las Cooperativas no Agropecuarias», op. cit., p. 1339.

27 A pesar de advertirse, en algunos casos, que no se permite a los asociados una participación activa en la dirección de la cooperativa (10\%), pues los socios no participan en la toma de decisiones, las que de hecho corresponden al presidente y tal vez alguno que otro socio más, por lo que el resto de los asociados tiene una representación formal en la Asamblea General, y carecen de posibilidades de participar en la toma de decisiones democráticas. 


\section{c) Relaciones de la empresa con el socio trabajador y sus familiares}

En el trabajo indagatorio realizado en las cooperativas, se pudo comprobar que para dar cumplimiento al objeto social de las mismas se establecían algunas actividades de apoyo, con evidente fin social $(25 \%)$, a lo interno de la cooperativa.

En el caso de la cooperativa de servicios contables, "Gesin», cuenta con el Programa «Fondo de Financiamiento», que permite otorgar a cada socio mil (\$1000.00 CUC) para la adquisición de equipos electrodomésticos, los que una vez adquiridos por el socio, y previa acreditación a la cooperativa de los justificantes de pago, esta procede al reembolso correspondiente. A su vez, la Cooperativa Reconstructora de Vehículos de La Habana decidió crear un fondo de entre 18 y 20 mil CUC para préstamos sin intereses a los socios ante determinadas eventualidades, 0 para premiar a los trabajadores más destacados. Para validar el principio cooperativista del aporte social la cooperativa atiende con precios moderados a los socios, propietarios de autos, jubilados o no, que no poseen la capacidad adquisitiva para contratar esos servicios ${ }^{28}$.

Interesante resaltar que, desde el año 2014, la Cooperativa Reconstructora de Vehículos realiza un Balance Social Cooperativo (BSCoop); y, aunque realmente constituye un informe contentivo de las principales acciones acometidas por la cooperativa en el ámbito social, y no trabaja con indicadores sistematizados, además, su registro en la contabilidad es el tradicional, constituye sin dudas, un salto cualitativo en la estrategia organizacional de la cooperativa, como contribución a sus buenas prácticas.

En general, las cooperativas en las cuales se realizó la investigación demostraron que existe especial interés en estas entidades por brindar a los socios servicios gastronómicos, constructivos y otros relacionados con adquirir bienes de consumo, para elevar la calidad de vida de los mismos y sus familias. Un número significativo de cooperativas (45\%), tanto del sector agropecuario, como no agropecuario, tienen implementados «cuentas de préstamo a los socios», sin intereses, lo que representa una ventaja para estos a la hora de poder utilizar determinados fondos previamente Por otra parte, un número importante de ellas $(60 \%)$ se preocupa por promover entre sus asociados el conocimiento y la educación de los principios y métodos cooperativos y difundirlos por todos los medios a su alcance. Igualmente, por implementar programas

28 Más ampliamente véase: Vuotto, M. y Verbeke, G., «Las cooperativas no agropecuarias en Cuba en el contexto de transformación de la Cuba de hoy. Desafíos actuales». XII Congreso Nacional de estudios del trabajo. Grupo Temático n. ${ }^{\circ} 11$ : Trabajo y autogestión en las organizaciones de la economía Social, Buenos Aires, Argentina, 2015, p. 2. 
de desarrollo que permitan aumentar el nivel técnico y profesional de los cooperativistas en materia de construcción, gastronomía, transporte y servicios contables, por solo mencionar algunos.

Es interés de las cooperativas cubanas, y así lo muestra el trabajo indagatorio, crear reservas para actividades socio-culturales con los socios y sus familias, las que cubren celebraciones y conmemoraciones, sobre todo en relación al aniversario de la cooperativa, el día del trabajador, cumpleaños colectivos, entre otros.

d) Relaciones de la empresa con los clientes, proveedores y el resto de los sujetos que integran la cadena de generación de valor de la empresa

El trabajo indagatorio dirigido a este aspecto permitió comprobar que las prácticas relacionales de la cooperativa se circunscriben a los procesos de negociación en la contratación con proveedores o suministradores y clientes, lo que constituye cumplimiento de las normas generales en materia de contratación económica, acompañado de acciones que realizan pocas organizaciones $(21 \%)$ de introducir actividades de estímulo moral y material a determinados suministradores por su contribución al desarrollo de la entidad (Cooperativa de servicios automotores «La Complaciente»), además de propiciar una interacción mayor entre el personal de la cooperativa y dichos sujetos en función de que se familiaricen con el desempeño del objeto social de la entidad o sus aspiraciones futuras.

Un ejemplo de buenas prácticas lo muestra Sancof, que a partir de febrero de 2017, decide invertir en el área de la comunicación, con la inclusión de un especialista dentro del organigrama empresarial. En función de esto: se diseñó un modelo integrativo de comunicación interna en la CNoA que permitió la optimización de la transmisión de información y el uso coherente y sistémico de los canales ya existentes y la creación de nuevos, por ejemplo, un boletín con circulación mensual, una revista con periodicidad cuatrimestral y la producción de materiales audiovisuales sobre la misión de SANCOF, su historia, resultados económicos y sociales. Esto trajo como resultado un mejor posicionamiento de la CNoA en la memoria de sus clientes, ganando prestigio y aumentando la cartera de negocios y confianza en la cooperativa 29 .

29 Montenegro Morales., M.: «Experiencias de buenas prácticas en la Cooperativa No Agropecuaria de Servicios de Construcción, Andamios y Cofres», op. cit., p. 116. 
e) Realización de acciones de mitigación de daño ambiental por las actividades de la cooperativa

En atención a este rubro, se pudo comprobar que solo se circunscriben a destinar un porciento de sus utilidades anuales a labores de este tipo y se atienen estrictamente a las normas técnicas y demás regulaciones que en materia ambiental emiten los organismos rectores. A pesar de que el $41 \%$ manifestó que sí se realizaban acciones de esta naturaleza, cuando se profundizaba en la indagación se pudo comprobar que existe muy poca iniciativa por parte de las cooperativas respecto de implementar mecanismos de precaución y/o prevención del daño ambiental. Esto debe comenzar con una conciencia ambiental por parte de cada uno de los trabajadores de la empresa para luego palpar los favorables, que no significa que algunas empresas no lo realicen, pero no son la mayoría, y lo más preocupante es que muchas veces los daños se vuelven irreversibles y las consecuencias son adversas para los habitantes de esa localidad o municipio.

Como ejemplo de buenas prácticas se expone el caso de Sancof, que al ser una cooperativa del sector constructivo, su actividad, de manera general, siempre genera residuos y contaminación. «Esta cooperativa cuenta con un Programa de compromiso medioambiental, cuya estrategia abarca desde una continua capacitación y superación del personal, a la participación activa para facilitar iniciativas que favorezcan el desarrollo socioeconómico de las comunidades vecinas. La cooperativa acometió en 2017 la reinauguración del puente peatonal Watkin, sobre el río Yumurí en la ciudad de Matanzas, que comunica el centro patrimonial con la barriada de Versalles. La obra permitió el rescate de un importante puente en una ciudad que se caracteriza por la cantidad de afluentes que convergen en su urbanización, mejoró la vialidad con altos niveles de seguridad para la vida de los transeúntes que a diario eligen el puente Watkin para disminuir distancias y tiempo entre dos puntos distantes de la ciudad, el saneamiento voluntario y sin costo alguno del río y los puntos de acceso al puente. Se demostró que una cooperativa de la construcción puede realizar obras proponiéndose minimizar el impacto sobre el medio ambiente» ${ }^{30}$.

A su vez, la cooperativa de reparación y mantenimiento de vehículos La Complaciente, manifiesta su compromiso con el medio ambiente con algunas iniciativas, entre las que destacan: la planificación

30 Montenegro Morales., M.: «Experiencias de buenas prácticas en la Cooperativa No Agropecuaria de Servicios de Construcción, Andamios y Cofres», op. cit., p. 118. 
y construcción de las nuevas instalaciones de oficinas de la cooperativa, respetando y favoreciendo al máximo la entrada de luz natural. Las antiguas instalaciones de la organización disponían de algunas oficinas en las que no había entrada de luz natural. El personal de la cooperativa sugirió que, en la planificación de las nuevas instalaciones, se tuviera en cuenta este aspecto. Con ello se ayuda a mejorar la calidad de vida de los socios y es una medida responsable con el medio ambiente.

En el sector agropecuario, varias cooperativas de la central provincia de Sancti Spíritus acometen proyectos que confirman que la aplicación de buenas prácticas eleva los rendimientos y encadenamientos productivos. En tres años, se construyeron en la Cooperativa de Producción Agropecuaria «Nueva Cuba» dos centros de inseminación de novillas, igual número de vaquerías y seis centros para el desarrollo de machos. Otro de los resultados que exhibe la entidad es la reforestación de 20 hectáreas con árboles frutales y declararse libre de áreas infestadas de aroma y marabú (plantas invasoras), después de limpiar más de 200 hectáreas contaminadas ${ }^{31}$.

Otra de las experiencias positivas tuvo lugar en la Cooperativa de Créditos y Servicios (CCS) «El Vaquerito», donde se mejoraron las condiciones ambientales de la cuenca hidrográfica del río Zaza. Por su parte, en la CCS «Julio Calviño», se comprobó la disminución del uso de agrotóxicos en la comunidad de Banao, mediante la introducción de insecticidas naturales como el árbol del Nim, cuyo aceite podrá ser utilizado para repeler plagas a partir de la próxima entrada en explotación de una minindustria.

Además, las cooperativas "Camilo Cienfuegos» y «Bienvenido Pardillo» contribuyen a la mitigación del cambio climático mediante la introducción de nuevas fuentes de energía renovable. Cada una de las entidades instaló 10 biodigestores, que utilizan los desechos sólidos de cerdos para generar biogás que utilizan en la cocción de alimentos. El proyecto de los biodigestores tiene también como uno de sus principales objetivos aliviar las labores de las mujeres rurales y la economía doméstica, al abaratar el costo del combustible, además de resultar ecológicamente limpio. La materia orgánica que va quedando funciona como fertilizante natural para las huertas, donde se cultivan hortalizas y frutales ${ }^{32}$.

31 Disponible en: https://www.ipscuba.net/economia/cooperativas-agropecuariasdel-centro-cubano-aplican-buenas-practicas/. Consultado el 20 de agosto de 2019.

32 Idem, p. 2. 
f) Vínculo de la empresa con la comunidad

«A la vez que atienden las necesidades de sus asociados, las cooperativas trabajan en pro del desarrollo sustentable de sus comunidades mediante políticas aprobadas por aquéllos.»

Este principio postula una idea bastante trascendental al promulgar que las cooperativas no solo atienden las necesidades de sus socios sino que además, tienen la responsabilidad de asegurar que el desarrollo económico, social, cultural y medio ambiental de sus comunidades sea sostenible, por medio de políticas aprobadas por sus propios miembros. Dicha responsabilidad es el resultado no de un acto de beneficencia hacia la comunidad, sino de tratar de coincidir con los de esta. En opinión de PIÑEIRO HARNECKER esto requiere el establecimiento de mecanismos de coordinación entre las cooperativas y los representantes de los intereses sociales ${ }^{33}$, a fin de que exista una materialización concreta del principio, y por medio de vías más factibles que posibiliten una mejor relación entre ambas partes.

El $70 \%$ de los encuestados manifestó que la cooperativa a la que pertenecen mantiene vínculos con la comunidad, aunque ninguna evalúa los impactos. Algunas plantearon (11\%) que tienen acciones comunitarias previstas pero sin cumplir, otras se refieren a las ya cumplidas como reparación de hogares de ancianos, bodegas, consultorios médicos etc., todo de forma gratuita o, en el caso de las cooperativas de construcción, cuando les facilitan los materiales aportan la mano de obra (29\%). El resto no tiene previstas acciones en la comunidad ni lo consideran relevante $(30 \%)$.

Las buenas prácticas en este ámbito abarcan acciones básicas de apadrinamiento o patrocinio, hasta otras más complejas que implican cierta permanencia y retroalimentación para el desarrollo de las actividades, como veremos más adelante.

Entre los ejemplos de buenas prácticas destacamos el caso de la cooperativa Reconstructora de Vehículos de La Habana, que apadrinó el Asilo Juan Lefont, del Municipio Cerro, realizando reparaciones de neveras y camas, la entrega de una bomba de agua y un freezer de almacenamiento. Asimismo, reparó 138 equipos, a precio acorde a las posibilidades de pago de las personas que los requirieron. Una de las líneas de trabajo incorporada está vinculada con la recuperación de am-

33 Piñeiro Harnecker, C.: «Las cooperativas en el nuevo modelo económico cubano», 2012, p. 5. Disponible a en www.anec.cu/.../2-LAS-COOPERATIVAS-NOAGROPECUARIAS-CO... Fecha de consulta: 20 de enero de 2017. 
bulancias y en este ámbito se reconstruyeron vehículos a un costo promedio de 3.833 CUC, contribuyendo de esta forma con el Sistema de Salud Pública del país ${ }^{34}$.

En el caso de la CNoA «Cocheros Oficina del Historiador», convocan a todos los coches y cocheros para realizar la inauguración del inicio del verano. Apadrinan, además, la escuela primaria que se encuentra cerca de la cooperativa y le prestan coches cuando realizan alguna actividad escolar como la Jornada Camilo-Che y Jornada Martiana. Por su parte la CNoA «La Complaciente», apadrina el circulo infantil «Amiguitos de Polonia», aledaño a la cooperativa; en ese sentido, realizan donativos para la celebración del inicio y fin de curso, acometieron la remodelación y embellecimiento de las áreas exteriores del círculo, con materiales reutilizables de goma, lo que permitió mejorar el pequeño parque del circulo; asimismo, contribuyeron con la compra de uniformes para todas las profesoras del Centro. La cooperativa tiene un elevado reconocimiento social en la localidad.

En el sector de los servicios contables, la cooperativa «Gesin» apadrina el hogar de niños sin amparo familiar "Luxon», enclavado en el municipio habanero de Arroyo Naranjo. Se ha realizado la entrega de equipos electrodomésticos como refrigeradores, cocinas, batidoras, equipos de cocción eléctricos, productos de aseo y otras, siempre en correspondencia con las necesidades del hogar. Interesante destacar, en este caso, que la cooperativa llega al hogar previa conciliación con el Ministerio de Educación, organismo al que acudieron en primera instancia, indagando en torno a donde colocar la ayuda. El Ministerio en ese momento indicó que debían contactar a la Dirección Municipal de Educación, y a partir de aquí se firma un «Acuerdo de Cooperación» entre la cooperativa y el organismo, en demostración de la necesidad de articular las intenciones entre los diferentes actores.

En el ámbito local, es notable que las cooperativas agropecuarias protagonizan palpables responsabilidades sociales en comunidades donde, por constituir zonas de difícil acceso, son ellas las que brindan un conjunto importante de servicios a sus asociados y a la comunidad. Por citar algunos: servicio de comedor y cafetería, servicio de venta de alimentos del agro, servicio de transporte laboral y social, servicios de

34 Vuotto, M. y Verbeke, G., "Las cooperativas no agropecuarias en Cuba en el contexto de transformación de la Cuba de hoy. Desafíos actuales». XII Congreso Nacional de estudios del trabajo. Grupo Temático N. ${ }^{\circ} 11$ : Trabajo y autogestión en las organizaciones de la economía Social, Buenos Aires, Argentina, 2015, p. 2. 
construcción y mantenimiento de viviendas y escuelas, servicios comunales, y servicio recreativo y cultural ${ }^{35}$.

En el caso de la cooperativa Reciclaje de Desechos de San José se reconoce la existencia de mejores condiciones para el desarrollo de una cultura de reciclaje en la comunidad, lo que favorece las acciones de responsabilidad social. Por otra parte, los indicadores económicos y productivos son favorables, se generan utilidades y existe capacidad económica para acciones de apoyo a la comunidad. Aunque, es menester destacar, que si bien se demuestra un compromiso con la comunidad, no se planifican acciones específicas para elevar la cultura de reciclaje, las pocas que realizan son espontánea y no se cuantifica económicamente lo aportado al desarrollo comunitario. Importante también señalar que, en este caso, los actores externos realizan una buena valoración sobre la gestión de la cooperativa y su impacto en la comunidad. Reconocen que lo anterior se logra por la buena preparación y capacitación de los socios, el nivel de ingresos obtenidos como resultado de la calidad en el servicio ${ }^{36}$.

El 33\% de los encuestados identificó, erróneamente, entre las acciones socialmente responsables que realiza la empresa «el pago del $1 \%$ como contribución al desarrollo local» ${ }^{37}$. No debe identificarse ello con la responsabilidad social de las cooperativas puesto que el aporte del $1 \%$ como contribución al desarrollo local es cumplir con una obligación legal a que viene obligada la cooperativa.

En resumen, del análisis presentado, podemos inferir que las problemáticas en torno al tema se clasifican en subjetivas y objetivas, las primeras asociadas, al desconocimiento de la temática y al entusiasmo de los cooperativistas que puedan ser más o menos proactivos en torno

35 Alfonso Alemán, J. L.: «Modelo de gestión de la Responsabilidad Social Cooperativa Directa. Estudio De Caso: Cooperativa De Producción Agropecuaria «Camilo Cienfuegos». Tesis Presentada en opción al grado científico de Doctor en Ciencias Económicas, Centro de Estudios sobre Desarrollo Cooperativo y Comunitario Facultad De Ciencias Económicas, Universidad de Pinar del Río, 2008, p. 81.

36 Soto Alemán, L.; Llanes Guerra, O. y González Ferrer, Y.: «El Balance Social Cooperativo en Cuba: Estado de la cuestión», op. cit., p. 62.

37 Cfr. Ley No. 113 de fecha 23 de julio de 2012, "Del sistema Tributario». Gaceta Oficial No. 53 Extraordinaria de 21 de noviembre de 2012.

«Art. 305.-Se establece una Contribución para el desarrollo sostenible de los municipios, que grava los ingresos por la comercialización de bienes o prestación de servicios, que obtengan las empresas, sociedades mercantiles y cooperativas, por sí mismas y por sus establecimientos en cada territorio.

Art. 306.-Son sujetos de esta Contribución, las empresas, las sociedades mercantiles de capital totalmente cubano y las cooperativas, por sus establecimientos, sin perjuicio del municipio en que estén enclavados». 
al tema; las segundas, más asociadas a las deficiencias que en el orden financiero enfrentan las cooperativas, que en muchos casos ven limitada su responsabilidad social, porque no encuentran resortes legales que fomenten estos comportamientos, sino que lo limitan.

Un aspecto conductor de todas las buenas prácticas expuestas es el énfasis emprendedor que tienen las cooperativas que los lleva a entender y desarrollar iniciativas sociales. Esto configura una cultura emprendedora en las cooperativas cubanas.

A pesar de los problemas descritos, es indudable que la RSE tiene enormes potencialidades en su dimensión interna ${ }^{38}$ y externa ${ }^{39}$, al tener manifestaciones concretas en esferas fundamentales como: la económica (compatibilizar la maximización de beneficios empresariales con la extensión del estado de bienestar), la social (vista en sus dos vertientes: externa, a partir de la articulación de intereses económicos con intereses sociales de la comunidad donde inserta su gestión el sujeto, e interna o laboral, mediante la defensa de los derechos de los trabajadores y la organización de la empresa y las políticas de empleo, seguridad y salud en el trabajo, capacitación y educación) y la medioambiental (mediante la prevención de riesgos ambientales, mitigación de daños, actitudes proactivas ante el cuidado del ambiente, formación y educación y consumo responsable). Con ella se persigue que el objetivo estratégico de la empresa no solo se enfoque en la generación de utilidades y el rendimiento económico en aras del cumplimiento de su objeto social, sino en la búsqueda de valor para todos los grupos de interés con los cuales interactúa. Cada una de estas aristas de la RSE deben estar acompañadas de un marco jurídico e institucional que no solo brinde legitimidad sino también seguridad jurídica.

En resumen, el catálogo de buenas prácticas expuesto ilustra una parte del caudal y la diversidad de las prácticas de las cooperativas cubanas en materia de RSCoop. Sin embargo, se constata que el concepto es aún poco conocido en el sector y que la mayoría de las buenas prácticas constituyen eventos aislados, carentes de sistematicidad, y no

38 Referente a las actividades y prácticas que cada organización debe contemplar en su gestión interna, en aspectos relacionados con la gestión de recursos humanos, la gestión ética y de buen gobierno, la calidad de los servicios, los sistemas de gestión implantada, y también los aspectos medioambientales vinculados. Esta vía abre una oportunidad para administrar el cambio y conciliar el desarrollo social con el aumento de la competitividad.

39 Comprende cómo la organización desarrolla sus competencias, fomentando e impulsando estos temas en su territorio, respetando los derechos de sus grupos de interés, el entorno, el medioambiente y la atención a las personas más desfavorecidas de su zona de actuación. 
están incorporados a la gestión económica de estas. Lógicamente, depende ello del escenario económico en el cual se desarrollan las cooperativas, los beneficios y los excedentes percibidos al finalizar el ejercicio fiscal son lo que motiva esta actuación.

\section{Ideas conclusivas}

La estricta observancia de los principios identitarios de una cooperativa debe constituir el faro que guie su actuación, sobre la base de procesos económicos eficientes y eficaces. La actualización del modelo económico cubano, con sus multicausalidades le otorga un papel esencial y complementario del sector empresarial estatal ${ }^{40}$; sin embargo, este encargo "económico» no puede desvirtuar las esencias de la figura, ya que su existencia como forma especial de asociación de personas con un objetivo común, las obliga a gestionar adecuadamente todas sus dimensiones y no únicamente las económicas.

Su misión social les viene por mandato del artículo 6 del Decreto Ley 366/19 que refrenda la responsabilidad social entre los principios identitarios que las impulsan, a lo interno con los socios y sus familiares, y a lo externo con las comunidades.

La aún débil percepción de la RSCoop, entronizada en el cumplimiento de los valores y principios identitarios de las cooperativas cubanas, distorsiona la esencia misma de esta organización como comunidad de personas al servicio de la sociedad. El análisis de los resultados en la muestra de cooperativas seleccionadas evidencia que aunque en una parte de ellas, se evidencia el ejercicio de buenas prácticas que fortalecen la gestión cooperativa, en general la RSCoop ha sido asumida de manera aislada y asistemática, como compromiso altruista de una parte de ellas, sin ser incorporada a las estrategias de gestión y planificación de la organización.

Las buenas prácticas cooperativas que les han elevado sus capacidades se manifiestan en los consensos logrados en la toma de decisiones, la constante preocupación por los procesos educacionales y formativos de los miembros de las CNoA, la cooperación entre cooperativas, y el vínculo con las comunidades, por solo mencionar algunos.

El compromiso de las cooperativas en asumir la RSCoop, y el posicionamiento adecuado para conseguirlo, no es suficiente. Resulta necesario poner en práctica esos compromisos mediante buenas prácti-

40 Cfr. numeral 2 de los Lineamientos..., op. cit., p. 10. 
cas cooperativas, informarlas, medirlas y evaluarlas. Para ello se pueden utilizar diferentes métodos, entre los que se encuentra el denominado Balance Social.

El BSCoop es una herramienta de gestión empresarial, de carácter público, ideal para la evaluación del cumplimiento de la responsabilidad social en un período determinado y frente a metas de desempeño preestablecidas por la organización con el objetivo de lograr una evaluación integral de los procesos ${ }^{41}$. Como herramienta de evaluación de la RSCoop facilita conjugar los aspectos económicos, sociales y medioambientales, y el logro de mayores beneficios no sólo para la cooperativa sino para la comunidad en la que opera.

El carácter público del BSCoop posibilita comunicar a los grupos de interés los resultados de la gestión social de la cooperativa, permitiría en el contexto cubano la comparación por las entidades verificadoras y auditores, del grado de cumplimiento de los principios cooperativos, estimulando las mejores cooperativas y desenmascarando las falsas que puedan crearse (carácter externo) y a las propias cooperativas rediseñar sus estrategias, establecer comparaciones con etapas precedentes, en fin, autoevaluarse (carácter interno). El espacio temporal coincidiría con el espacio del balance financiero, año fiscal, y respondería esta herramienta a estrategias previamente diseñadas y acordadas por todos los socios.

Finalmente, tener en cuenta, como premisa importante para la gestión social de las cooperativas, el reconocimiento y apoyo del Estado a la acción social directa de estas organizaciones. El Estado puede promover buenas prácticas cooperativas mediante exenciones fiscales y bonificaciones para aquellas cooperativas que obtienen resultados satisfactorios, así como el financiamiento a programas de desarrollo de estas prácticas. No debe pensarse en una responsabilidad social limitada únicamente a quienes deben llevarla a cabo, el Estado tiene un rol esencial y en este sentido puede establecer un marco referencial mínimo para la promoción y estímulo de acciones responsables.

En general, las medidas de fomento hacia el sector cooperativo, armonizarían con la Recomendación sobre la promoción de las Cooperativas 2002 de la Organización Internacional del Trabajo (R. 193). En tal

41 Para más información sobre los presupuestos teórico-jurídicos que pueden informar la regulación del Balance Social Cooperativo en Cuba, se puede consultar: Soto Alemán, L.: «Notas en pos de la regulación jurídica del balance social cooperativo en Cuba», en el Boletín de la Asociación Internacional de Derecho Cooperativo, Núm. 51/2017. Bilbao, págs. 317-344. 
sentido los Estados deben adoptar medidas tendentes a promover el potencial de las cooperativas con el fin de ayudarlas a ellas y a sus asociados a crear y desarrollar actividades generadoras de ingresos y empleo decente, fomentar el conocimiento de los valores y principios del cooperativismo, así como de sus ventajas y beneficios mediante la educación y la formación ${ }^{42}$.

\section{Bibliografía}

AlbuQuerque LloRens, Francisco: «Desarrollo económico local y descentralización en América Latina», Revista de la CEPAL, No. 82, 2004.

Alfonso Alemán, J.L.: «Modelo de gestión de la Responsabilidad Social Cooperativa Directa. Estudio De Caso: Cooperativa De Producción Agropecuaria «Camilo Cienfuegos». Tesis Presentada en opción al grado científico de Doctor en Ciencias Económicas, Centro de Estudios sobre Desarrollo Cooperativo y Comunitario Facultad De Ciencias Económicas, Universidad de Pinar del Río, 2008.

Alfonso Alemán, J.L.; Rivera-Rodríguez, C. A.; Labrador-Machín, O.: «Responsabilidad social empresarial. sus particularidades en las empresas cooperativas», Revista Avances, CIGET Pinar del Rio, Vol. 11, n. ${ }^{\circ}$ 1, marzo 2009.

Bastidas Delgado, O.: "La especificidad cooperativa», Universidad Central de Venezuela, Caracas, 2004.

FreEmAn, R.E.: Strategic management: a stakeholders approach, en Pitman press, Boston, 1984.

Montenegro Morales, M.: «Experiencias de buenas prácticas en la Cooperativa No Agropecuaria de Servicios de Construcción, Andamios y Cofres», COODES, Revista de Cooperativismo y Desarrollo, Volumen n. ${ }^{\circ} 6, \mathrm{n} .{ }^{\circ} 1$, enerojunio, 2018.

PINEEIRO HARNECKER, C.: "Las cooperativas en el nuevo modelo económico cubano», 2012, p. 5. Disponible a en www.anec.cu/.../2-LAS-COOPERATIVASNO-AGROPECUARIAS-CO... Fecha de consulta: 20 de enero de 2017.

Rivera-Rodríguez, C.A.; Labrador-Machín, O.; Alfonso-Alemán, J. L: "Retos del cooperativismo como alternativa de desarrollo ante la crisis global. Su papel en el modelo económico cubano». En Piñeiro HarneCKer, C. (comp.): Cooperativas y Socialismo: una mirada desde Cuba, Editorial Caminos, la Habana, 2011.

Rodríguez Musa, O.: La Cooperativa como figura jurídica. Editorial DYKINSON, S.L., Madrid 2011.

42 Vid. Conferencia General de la Organización Internacional del Trabajo: Convocada en Ginebra por el Consejo de Administración de la OIT, 3 de junio de 2002, disponible en: $w w w$.ilo.org/dyn/normlex/es/f?p=1000:12100:0::NO::P12100, Fecha de consulta: 16 de febrero de 2019. 
Rodríguez Musa, O.: Presupuestos Teóricos para el redimensionamiento constitucional de la cooperativa en Cuba, Tesis en opción al grado científico de Doctor en Ciencias Jurídicas, La Habana, 2016.

Soto Alemán, L.: Presupuestos Teórico-Jurídicos del Balance Social Cooperativo en Cuba, Tesis en opción al grado científico de Master en Derecho de la Economía, La Habana, 2016.

Soto Alemán, L.; llanes Guerra, O. y GonzÁlez Ferrer, Y.: «El Balance Social Cooperativo en Cuba: Estado de la cuestión». En SCHUJMAN, M.; ITURRASPE Oviedo, F.; Fajardo García, I. y Chaves Gaudio, R. (coords.): La participación de los asociados en las entidades de la economía social y solidaria. Cooperativas de trabajo asociado. Autogestión. Carta del trabajador autogestionario, 1. ${ }^{\text {a }}$ ed. Rosario: UNR Editora. Editorial de la Universidad Nacional de Rosario, 2019.

VACA AcOSTA et al.: "Análisis de la Responsabilidad Social Corporativa desde tres enfoques; stakeholders, capital intelectual y teoría institucional», Revista Conocimiento, innovación y emprendedores; camino al futuro. Vol. 3. Universidad de La Rioja, España. Disponible en: <http//: dialnet. unirioja.es/descarga/articulo/2234835.pdf>. Fecha de consulta: 5 de mayo de 2016.

VUOtTo, M. y VerbeKE, G., "Las cooperativas no agropecuarias en Cuba en el contexto de transformación de la Cuba de hoy. Desafíos actuales». XII Congreso Nacional de estudios del trabajo. Grupo Temático n. ${ }^{\circ} 11$ : Trabajo y autogestión en las organizaciones de la economía Social, Buenos Aires, Argentina, 2015.

\section{Otros documentos}

Alianza CoOperativa Internacional: «Declaración sobre la Identidad Cooperativa». Disponible en: www.idelcoop.org.ar/sites/default/files/revista/articulos/pdf/...Fecha de consulta: 20 de octubre de 2014.

Organización INTERNACIONAL de NORMalZación-Norma InTERnACIONAL ISO 26000 , Guía de Responsabilidad Social: Primera Edición, Ginebra, 2010. Disponible en http://www.iso.org/iso/iso_26000_project_overview-es.pdf. Fecha de consulta: 12 de noviembre de 2016 .

Organización Internacional del Trabajo: Conferencia General convocada en Ginebra por el Consejo de Administración de la OIT, 3 de junio de 2002, disponible en: www.ilo.org/dyn/normlex/es/f?p=1000:12100:0::NO::P12100, Fecha de consulta: 16 de febrero de 2019.

Partido Comunista de Cuba (VII Congreso): Actualización de los Lineamientos de la Política Económica y Social del Partido y la Revolución para el período 2016-2021, abril de 2016. Recuperado de http://www.cubadebate. cu/especiales/2016/09/13/vea-el-texto-integro-de-la-actualizacion-de-loslineamientos-para-el-periodo-2016-2021-pdf/. Consultado el 2 de octubre de 2016. 


\section{Legislación}

Constitución de la República de Cuba. Gaceta Oficial Extraordinaria No. 5, de 10 de abril de 2019.

DeCReto Ley No. 365. «De las Cooperativas Agropecuarias». Gaceta Oficial Ordinaria No. 37, de 24 de mayo de 2019.

DeCreto Ley No. 366, «De las Cooperativas no Agropecuarias». Gaceta Oficial Ordinaria No. 63, de 30 de agosto de 2019.

DeCRETO No. 354. «Reglamento de las Cooperativas Agropecuarias Gaceta Oficial Ordinaria No. 37, de 24 de mayo de 2019.

DeCRETo No. 356. «Reglamento de las Cooperativas no Agropecuarias. Gaceta Oficial Ordinaria No. 63, de 30 de agosto de 2019. 


\section{Derechos de autor}

http://dec.revistas.deusto.es/

La revista Deusto Estudios Cooperativos es una revista de acceso abierto lo que significa que es de libre acceso en su integridad inmediatamente después de la publicación de cada número. Se permite su lectura, la búsqueda, descarga, distribución y reutilización legal en cualquier tipo de soporte sólo para fines no comerciales y según lo previsto por la ley; sin la previa autorización de la Editorial (Universidad de Deusto) o el autor, siempre que la obra original sea debidamente citada (número, año, páginas y DOI si procede) y cualquier cambio en el original esté claramente indicado.

\section{Copyright}

The Deusto Journal of Cooperative Studies is an Open Access journal which means that it is free for full and immediate access, reading, search, download, distribution, and lawful reuse in any medium only for non-commercial purposes, without prior permission from the Publisher or the author; provided the original work is properly cited and any changes to the original are clearly indicated. 